phys. stat. sol. (b) 81,479 (1977)

Subject classification: 13.5 .1 and $20.1 ; 22.9$

Department of Chemistry, The University of Michigan, Ann Arbor ${ }^{1}$ )

\title{
Molecular Exciton Cluster States and Spectra with Application to Benzene ${ }^{2}$ )
}

By

\author{
J. Hoshen and R. Kopelman
}

A cluster expansion is introduced for the determination of the optical absorption properties of cluster states in substitutionally disordered molecular crystals. This method is based on the Green's function expansion in terms of the localized cluster states in the energy region of the guest subband. The general features of the method are illustrated by calculations for a simple two-dimensional molecular crystal. The physical parameters for the calculation are based on the $v_{11}$ benzene vibrational exciton. Both polarized and unpolarized cluster optical absorptions display strong asymmetries, with respect to the isolated guest spectral line. Calculations are performed on the monomer, dimer, trimer, and tetramers (three kinds) of the benzene crystal ac-plane.

Es wird eine Clusterentwicklung für die Bestimmung der optischen Absorptionseigenschaften der Clusterzustände in substitutionsfehlgeordneten Molekularkristallen eingeführt. Diese Methode beruht auf der Entwicklung der Greenschen Funktion nach den lokalisierten Clusterzuständen im Energiebereich der Gast-Subbanden. Das allgemeine Vorgehen dieser Methode wird durch Rechnungen für einen simplen zweidimensionalen Molekülkristall illustriert. Die physikalischen Parameter für die Rechnungen basieren auf dem Benzen-v $\boldsymbol{v}_{11}$-Schwingungsexziton. Sowohl die polarisierte als auch die unpolarisierte optische Clusterabsorption zeigt starke Asymmetrie bezüglich der isolierten Gast-Spektrallinie. Rechnungen werden für die Monomere, Dimere, Trimere und Tetramere (drei Arten) der Benzenkristall- $\alpha c$-Ebene durchgeführt.

\section{Introduction}

The understanding of the optical properties of isotopic mixed molecular crystals has improved appreciably in recent years $[1,2]$. The introduction of the Coherent Potential Approximation (CPA) and the moment expansion method for isotopically mixed molecular crystals provided a useful tool for the determination of the optical spectra of these crystals for varying concentrations of the isotopic component [ 1 to 5]. However, the CPA and moment methods fail to provide information on the fine structure of the density-of-states function and the optical absorption spectra [ 6 to 8]. The fine structure in the density-of-states function and in the optical spectra is generated by the presence of clusters of guest ${ }^{3}$ ) molecules in the mixed crystal. The effect of these clusters is pronounced for guest molecules below the percolation concentration $[2,6,9]$ for large guest perturbation strength, relative to the exciton bandwidth.

In this paper we shall extend the cluster expansion method used by Hoshen and Jortner [9] for the density-of-states of substitutionally disordered crystals. In the previous work, clusters in a one-dimensional system were investigated. In the present work we shall explore a two-dimensional crystal structure topology applicable to some benzene crystal excited states. In Section 2 the cluster method is expanded to include crystals with several molecules per unit cell. The optical properties related to these

1) Ann Arbor, Michigan 48 109, USA.

2) Supported by NSF Grant DMR75-07832 A01 and NIH Grant NS08116-08.

3) "guest" is the definition of any one of the two substitutional components depending on convenience - usually that on which the eigenstate of interest is preferentially localized on. 
clusters are determined in Section 3. In Section 4 the cluster method is applied to clusters in two-dimensional systems. Numerical data are given in Section $\mathbf{5}$ for a vibrational exciton in a substitutionally disordered benzene crystal. These numerical results compare well with available experimental data.

\section{A Cluster Expansion Method for the Density of States Optical Properties}

Hoshen and Jortner [9] derived an expansion for the portion of the density-of-states function generated by the guest clusters embedded in the host crystal containing one molecule per primitive unit cell. The same formulas can be applied with slight modifications to crystals containing several molecules per unit cell. We shall consider an exciton state for the pure crystal derived from a single molecular state

$$
|\boldsymbol{k}, \nu\rangle=\sum_{i=1}^{\sigma} B_{i v}(\boldsymbol{k})|\boldsymbol{k}, i\rangle \text {. }
$$

In (2.1) $\boldsymbol{k}$ denotes the wave vector, $\sigma$ the order of the interchange group [10] and $\nu$ the exciton branch where $v=1,2, \ldots, \sigma$. The $B_{i v}(\boldsymbol{k})$ coefficients of the "one site exciton wave function", $|\boldsymbol{k}, i\rangle$, corresponding to site $i$ in the unit cell, can be determined by diagonalizing the matrix $L(\boldsymbol{k})$ whose elements are given by

$$
L_{i j}(\boldsymbol{k})=\left\langle\boldsymbol{k}, i\left|\hat{H}^{\mathbf{0}}\right| \boldsymbol{k}, j\right\rangle,
$$

where $\hat{H}^{0}$ is the Hamiltonian of the pure host crystal. The one site exciton wavefunctions are given in terms of the localized excitations $|n, i\rangle$ :

$$
|\boldsymbol{k}, i\rangle=(N \boldsymbol{\sigma})^{-1 / 2} \sum_{n} \mathrm{e}^{i \boldsymbol{k}\left(\boldsymbol{R}_{n}+\boldsymbol{S}_{i}\right)}|n, i\rangle .
$$

$\boldsymbol{S}_{i}$ denotes the position of the $i$-th molecule in the $n$-th unit cell and $\boldsymbol{R}_{n}$ denotes the position of the origin on the $n$-th unit cell. $N$ is the number of unit cells.

The basic expression for the density of states of the mixed crystal is $[2,11]$

$$
\varrho(E)=\frac{1}{\pi N \sigma} \operatorname{Im} \operatorname{Tr} G(E)=\varrho^{0}(E)+\frac{1}{\pi N \sigma} \operatorname{Im}\left[\frac{\mathrm{d} \ln D(E)}{\mathrm{d} E}\right],
$$

where $\varrho^{0}(E)$ is the density of states per molecule of the pure host crystal, $G$ is the disordered (perturbed) crystal Green's function and $D(E)$ is a determinant given by

$$
D(E)=\left|I-G^{0}(E) V\right| \text {. }
$$

$G^{0}(E)$ is the Green's function matrix for the unperturbed crystal (pure crystal) with its elements being given by

$$
G_{n i ; m j}^{0}(E)=\left\langle n, i\left|\hat{G}^{0}(E)\right| m, j\right\rangle,
$$

where $\hat{G}^{0}$ is the pure crystal Green's function operator. (The matrix elements $(2.4 \mathrm{~b}$ ) are sometimes called superexchange interactions.) The perturbed matrix elements are given by:

$$
\langle n, i|V| m, j\rangle=\eta \Delta \delta_{n m} \delta_{i j},
$$

where $\eta=1$ when the site $n i$ is occupied by a guest molecule and $\eta=0$ when the site $n i$ is occupied by a host molecule. $\Delta$ is the difference between the excitation energy of a single guest molecule and a single host molecule. (2.4) can be further simplified by partitioning the $G^{0}(E)$ and $V$ matrices into four submatrices.

$G^{0}(E)$ is given as [9]

$$
G^{0}(E)=\left(\begin{array}{ll}
G_{\mathrm{gg}}^{0}(E) & G_{\mathrm{gh}}^{0}(E) \\
G_{\mathrm{hg}}^{0} & G_{\mathrm{hh}}^{0}(E)
\end{array}\right)
$$


$G_{\mathrm{gg}}^{0}$ includes Green's function matrix elements connecting guest molecules only, whereas $G_{\mathrm{hh}}^{0}(E)$ connects host molecules only. $G_{\mathrm{gh}}^{0}(E)$ and $G_{\mathrm{hg}}^{0}(E)$ include elements connecting guest and host molecules only.

The dimensions of the $G_{\mathrm{gg}}^{0}(E)$ and $G_{\mathrm{hh}}^{0}(E)$ square submatrices are $C \sigma N$ and $(1-C) \sigma N$, respectively. $C$ denotes the guest concentration. Similarly $V$ is constructed:

$$
V=\left(\begin{array}{cc}
V_{\mathrm{g}} & 0 \\
O & O_{\mathrm{h}}
\end{array}\right) .
$$

$V_{\mathrm{g}}$ is a diagonal matrix of the same order of $G_{\mathrm{gg}}^{0}(E)$ :

$$
V_{\mathrm{g}}=\Delta I_{\mathrm{g}} \text {. }
$$

$O_{\mathrm{h}}$ is a null matrix of the order of $G_{\mathrm{hh}}^{0}(E)$, and $I_{\mathrm{g}}$ is the identity matrix.

Utilizing (2.5), (2.6) and $(2.7),(2.4 \mathrm{a})$ can be recast:

$$
D(E)=\operatorname{det}\left|I_{\mathrm{g}}-\mathcal{A}_{\mathrm{gg}}^{0}(E)\right| \text {. }
$$

The determinant of (2.8) can be further reduced if we assume [9] that the superexchange interactions $[6,2]$ are non-zero between only a finite number of molecules. This approximation is valid when $|\Delta|$ is large relative to the bandwidth [9]. Under the above assumption $G_{\mathrm{gg}}^{0}(E)$ can be represented in a quasidiagonal form [9], where each member of the submatrices along the diagonal represents a particular cluster of guest molecules. There will be $N_{n \alpha}$ such submatrices $G_{n \alpha}^{0}(E)$ of order $n$ and shape $\alpha$. $G_{\mathrm{gg}}^{0}(E)$ can be represented as

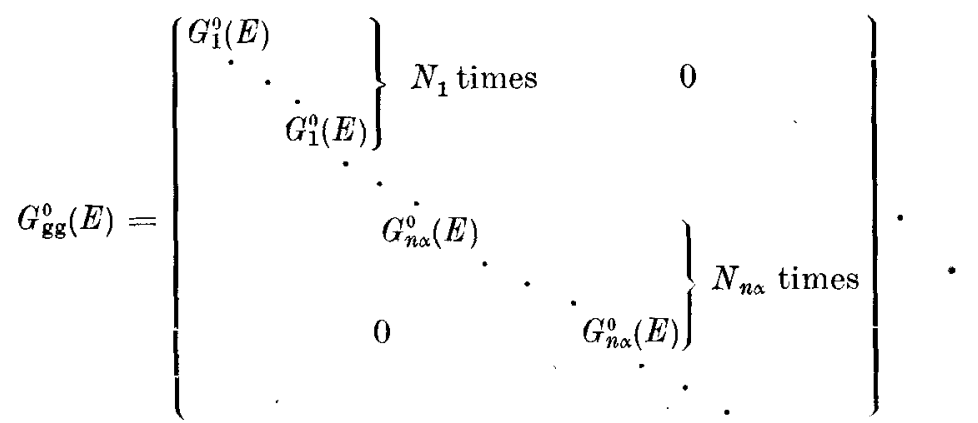

It should be noted that the cluster definition depends on the perturbation strength $\Delta$. Only for large $|\Delta|$ relative to the bandwidth can the clusters be defined in the classical sense in terms of the direct interactions rather than the superexchange interactions. $D(E)$ is given by

$$
D(E)=\Pi_{n \alpha}\left[D_{n \alpha}(E)\right]^{N_{n \alpha}}
$$

where $D_{n \alpha}$ has the form

$$
D_{n \alpha}(E)=\left|I_{n}-\Delta G_{n \alpha}^{0}(E)\right| .
$$

The density of states for the guest band $\varrho^{\mathrm{g}}$ is given by [9]

$$
\varrho^{\mathrm{g}}(E)=\sum_{n \alpha} P_{n \alpha} \sum_{m} \delta\left(E-E_{m}(n \alpha)\right) \text {. }
$$

$E_{m}(n \alpha)$ is the $m$-th root of $D_{n \alpha}(E)$, and $P_{n \alpha}$ is the probability of locating cluster $n \alpha$ in the crystal. 


\section{Optical Properties}

The dipole strength per unit of energy is given by $[5,2]$

$$
J(E)=\frac{1}{\pi} \operatorname{Im}\langle 0|\hat{\mu} \hat{G} \hat{\mu}| 0\rangle,
$$

where $|0\rangle$ is the ground state wave function, $G$ is the crystal Green's operator function and $\hat{\mu}$ is the dipole operator.

$\hat{G}$ can be given:

$$
\widehat{G}(E)=\hat{G}^{\mathbf{0}}(E)+\hat{G}^{\mathbf{1}}(E),
$$

where $\hat{G}^{0}$ and $\hat{G}^{1}$ represent the unperturbed part (pure host crystal) and perturbed part of the Green's function $\hat{G}$, respectively.

Our concern will be only with $\hat{G}^{\mathbf{1}}$, since $\hat{G}^{1}$ contains the guest clusters contributions. It is given by:

$$
\hat{G}^{1}=\hat{G}^{0} \hat{V}\left(\hat{I}-\hat{G}^{0} \hat{V}\right)^{-1} \hat{G}^{0} \text {. }
$$

For the guest region of $E$ we can define

$$
T(E)=\frac{1}{\pi}\left\langle 0\left|\hat{\mu} \hat{G}^{1} \hat{\mu}\right| 0\right\rangle .
$$

Applying the projection operator

$$
\hat{I}=\sum_{v^{\prime}=1}^{\sigma} \sum_{\boldsymbol{k}}\left|\boldsymbol{k}, \boldsymbol{v}^{\prime}\right\rangle\left\langle\boldsymbol{k}, \boldsymbol{v}^{\prime}\right|
$$

twice on $T(E), T(E)$ can be recast for the $\nu$-th exciton branch in the form:

$$
\begin{aligned}
T^{v}(E) & =\frac{1}{\pi}|\langle 0|\hat{\mu}| u, v\rangle|^{2} \sum_{k^{\prime} v^{\prime}}\left\langle 0 v\left|\hat{G}^{0}\right| k^{\prime} v^{\prime}\right\rangle \otimes \\
\otimes & \sum_{k^{\prime \prime} v^{\prime \prime}}\left\langle k^{\prime} v^{\prime}\left|\hat{V}\left(I-\hat{G}^{0} \hat{V}\right)^{-1}\right| k^{\prime \prime} v^{\prime \prime}\right\rangle\left\langle k^{\prime \prime}, v^{\prime}\left|G^{0}\right| u, v\right\rangle .
\end{aligned}
$$

$T^{y}(E)$ can now be represented by

$$
T^{v}(E)=\left|\mu^{v}\right|^{2}\left[E-E^{v}(0)\right]^{-2} S^{v}(E),
$$

where $E^{\nu}(0)$ denotes the eigenvalue for $|\boldsymbol{k}=0, v\rangle, \mu^{\nu}$ is given by

$$
\mu^{v}=\langle 0|\mu| 0, v\rangle
$$

and $S^{v}(E)$ has the form

$$
\begin{aligned}
S^{\nu}(E) & =\frac{1}{\pi}\left\langle 0, \nu\left|\hat{V}\left(I-\hat{G}^{0} \hat{V}\right)^{-1}\right| 0, v\right\rangle== \\
& =(\sigma N \pi)^{-1} \sum_{l} \sum_{i} \sum_{m} \sum_{j} B_{i \nu}^{*}(0) B_{j \nu}(0)\left\langle l i\left|\hat{V}\left(\hat{I}-\hat{G}_{0} \hat{V}\right)^{-1}\right| m j\right\rangle .
\end{aligned}
$$

Let us define a matrix $W$ such that

$$
W=V\left(I-G^{0} V\right)^{-1} \text {. }
$$

Utilizing (2.5), (2.6) and (2.7) the matrix $W$ can be written in the form

$$
W=A\left(\begin{array}{cc}
\left(I_{\mathrm{g}}-\Delta G_{\mathrm{gg}}^{0}\right)^{-1} & O \\
O & O_{\mathrm{h}}
\end{array}\right) \text {. }
$$


Since the summation over $l i$ and $m j$ in (3.9) is carried only over guest molecules only the $W_{\mathrm{gg}}$ elements in $(3.11)$ are not zero. If the $G_{\mathrm{gg}}^{0}(E)$ is arranged according to $(2.9), S^{v}$ can be written in the form

$$
S^{\nu}(E)=\frac{\Delta}{\pi} \sum_{n \alpha} \frac{P_{n \alpha} M_{n \alpha}^{(v)}(E)}{D_{n \alpha}(E)},
$$

where $M_{n \alpha}^{(\nu)}$ is given by

$$
M_{n \alpha}^{(\nu)}(E)=\sum_{l i \in Z_{n \alpha}} \sum_{m j \in Z_{n \alpha}} \operatorname{Minor}\left\langle l i\left|\left(I-G^{0} V\right)^{-1}\right| m j\right\rangle \otimes B_{i v}^{*}(0) B_{j v}(0)(-1)^{l+m+i+j} .
$$

$Z_{n \alpha}$ denotes a set of molecules belonging to a guest cluster of type $n \alpha$, and $P_{n \alpha}$ is the probability of locating such a cluster in the crystal. Then contributions to the imaginary part of $S^{\nu}(E)$ are from the zeros of $D_{n \alpha}(E)$. However, forbidden transitions do not contribute to the imaginary part of $S^{v}(E)$. Some of the $E_{m}(n \alpha)$ roots of $D_{n \alpha}(E)$ correspond to forbidden transitions. Common factors corresponding to these roots can cancel out in the ratio $M_{n \alpha}^{(\nu)}(E) / D_{n \alpha}(E)$. In this case the following expression can be written for the reduced form of $M_{n \alpha}^{v}(E) / D_{n \alpha}(E)$ :

$$
\frac{M_{n \alpha}^{(v, \mathrm{r})}}{D_{n \alpha}^{(v, \mathrm{r})}}=\frac{M_{n \alpha}^{(v)}(E)}{D_{n \alpha}(E)}
$$

where $\mathbf{r}$ represents reduced terms.

Utilizing the identity

$$
\operatorname{Im} \frac{1}{x}=\pi \delta(x)
$$

we obtain for $\operatorname{Im} S^{\nu}(E)$

$$
\operatorname{Im} S^{\nu}(E)=\sum_{n \alpha} \frac{P_{n \alpha} M_{n \alpha}^{(\nu, \mathrm{r})}(E)}{\left[D_{n \alpha}^{(v, \mathrm{r})}(E)\right]^{\prime}} \sum_{m} \delta\left[E-E_{m}(n \alpha)\right] .
$$

The summation over $m$ is carried over the roots of $D_{n \alpha}^{(v, \mathbf{r})}(E) .\left[D_{n \alpha}^{(v, \mathbf{r})}(E)\right]^{\prime}$ denotes a derivative with respect to $E$,

$$
\left[D_{n \alpha}^{(v, \mathrm{r})}(E)\right]^{\prime}=\frac{\mathrm{d} D_{n \alpha}^{(v, \mathrm{r})}(E)}{\mathrm{d} E}
$$

\section{The Benzene Crystal Green's Functions}

The Benzene crystal belongs to the $D_{2 \mathrm{~h}}^{15}$ space group and contains four interchange equivalent molecules per unit cell [10]; thus there are four branches for the exciton band $[2,10,12$ to 16$], \sigma=4$ in (2.1) and the four site exciton wave functions $|k, i\rangle$ are given by (2.3). The site vectors [17] $S_{v}$ of (2.3) are

and

$$
\begin{aligned}
& \mathbf{S}_{1}=\boldsymbol{O}, \\
& \mathbf{S}_{2}=\frac{1}{2}\left(a_{1}+a_{2}\right), \\
& \mathbf{S}_{3}=\frac{1}{2}\left(a_{2}+a_{3}\right),
\end{aligned}
$$

$$
S_{4}=\frac{1}{2}\left(a_{3}+a_{1}\right),
$$

where $\boldsymbol{a}_{1}, \boldsymbol{a}_{2}$ and $\boldsymbol{a}_{3}$ are the three primitive lattice vectors. The Green's function matrix elements for the pure host crystal are given by (see $(2.4 \mathrm{~b})$ )

$$
G_{n i, m j}^{0}(E)=\frac{1}{4 N} \sum_{y=1}^{4} \sum_{\boldsymbol{k}} \frac{B_{i v}^{*}(\boldsymbol{k}) B_{i v}(\boldsymbol{k}) \mathrm{e}^{i \boldsymbol{k}\left(\boldsymbol{R}_{m}-\boldsymbol{R}_{n}+\boldsymbol{S}_{j}-\mathbf{S}_{i}\right)}}{E-\overline{E^{\nu}}(\boldsymbol{k})}
$$


where $E^{\nu}(\boldsymbol{k})$ are the eigen-values of the pure crystal Hamiltonian $H^{\mathbf{0}}$, and where the coefficients $S_{j l}$ determine the vector $\mathbf{S}_{j}$ (see (4.1))

$$
\mathbf{S}_{j}=S_{j 1} \boldsymbol{a}_{1}+S_{j 2} \boldsymbol{a}_{2}+S_{j 3} \boldsymbol{a}_{3}
$$

For the benzene crystal, in the restricted Frenkel limit [17], and replacing the summation in (4.2) by integration, we get

$$
\begin{aligned}
& G_{n i, m j}^{0}(E)=\frac{1}{32 \pi^{2}} \sum_{v=1}^{4} B_{i v} B_{j v} \int_{-\pi}^{\pi} \mathrm{d} y^{\prime} \exp \left[i y^{\prime}\left(m_{2}-n_{\mathrm{i}}+S_{j 2}-S_{i 2}\right) \otimes\right. \\
& \times \int_{-\pi}^{\pi} \mathrm{d} z^{\prime} \exp \left[i z^{\prime}\left(m_{3}-n_{3}+S_{j 3}-S_{i 3}\right)\right] \int_{-\pi}^{\pi} \frac{\mathrm{d} x^{\prime} \exp \left[i x^{\prime}\left(m_{1}-n_{1}+S_{j 1}-S_{i 1}\right)\right]}{E-E^{v}\left(x^{\prime}, y^{\prime}, z^{\prime}\right)} .
\end{aligned}
$$

It should be noted that here [17] $B_{i v}= \pm 1$.

In the present work we shall impose further restrictions on the inter-molecular interaction parameters. We shall assume that there are only three sets of non-zero interactions, corresponding to nearest neighbor interchange equivalent molecules. These are

$$
\begin{aligned}
A & =\left\langle 0,1\left|\hat{H}^{0}\right| 0,2\right\rangle, \\
B & =\left\langle 0,1\left|\hat{H}^{0}\right| 0,3\right\rangle, \\
C & =\left\langle 0,1\left|\hat{H}^{0}\right| 0,4\right\rangle .
\end{aligned}
$$

Thus the $L(\boldsymbol{k})$ matrix elements are given by

$$
\begin{aligned}
& L_{11}(\boldsymbol{k})=0, \\
& L_{12}(\boldsymbol{k})=4 A \cos \left(\frac{\boldsymbol{k} \boldsymbol{a}_{\mathbf{1}}}{2}\right) \cos \left(\frac{\boldsymbol{k} \boldsymbol{a}_{2}}{2}\right), \\
& L_{13}(\boldsymbol{k})=4 B \cos \left(\frac{\boldsymbol{k} \boldsymbol{a}_{2}}{2}\right) \cos \left(\frac{\boldsymbol{k} \boldsymbol{a}_{3}}{2}\right), \\
& L_{\mathbf{1 4}}(\boldsymbol{k})=4 C \cos \left(\frac{\boldsymbol{k} \boldsymbol{a}_{3}}{2}\right) \cos \left(\frac{\boldsymbol{k} \boldsymbol{a}_{1}}{2}\right) .
\end{aligned}
$$

$E^{v}\left(x^{\prime}, y^{\prime}, z^{\prime}\right)$ has the form

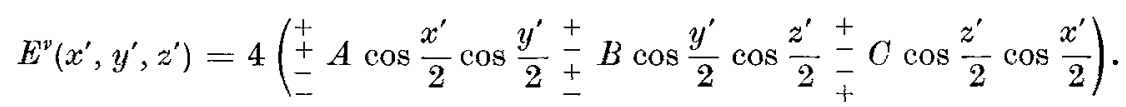

(4.4) can be rewritten for the parameters (4.5) to (4.12) as:

$$
\begin{aligned}
& G_{n i m j}^{0}(E)=\frac{1}{4 \pi^{3}} \int_{-\pi / 2}^{\pi / 2} \mathrm{~d} y \exp \left[2 i y\left(m_{2}-n_{2}+S_{j 2}-S_{i 2}\right)\right] \times \\
& \times \int_{-\pi}^{\pi} \mathrm{d} z \exp \left[2 i z\left(m_{3}-n_{3}+S_{j 3}-S_{i 3}\right) I \int_{-\pi}^{\pi} \frac{\mathrm{d} x \exp \left[2 i x\left(m_{1}-n_{1}+S_{j 1}-S_{i 1}\right)\right]}{E-h(x, y, z)},\right.
\end{aligned}
$$

where $h(x, y, z)$ is given by

$$
h(x, y, z) \equiv E^{1}(x, y, z)=4 A \cos x \cos y+4 B \cos y \cos z+4 C \cos z \cos x
$$


The Green's functions matrix elements corresponding to the three nearest neighbor interchange equivalent molecules can be expressed in the form

$$
\begin{aligned}
& g_{0}(E)=G_{01 ; 01}^{0}(E)=\frac{1}{4 \pi^{3}} \int_{-\pi / 2}^{\pi / 2} \mathrm{~d} y \int_{-\pi}^{\pi} \mathrm{d} z \int_{-\pi}^{\pi} \frac{\mathrm{d} x}{E-h(x, y, z)}, \\
& g_{1}(E)=G_{01 ; 02}^{0}(E)=\frac{1}{4 \pi^{3}} \int_{-\pi / 2}^{\pi / 2} \mathrm{~d} y \mathrm{e}^{i y} \int_{-\pi}^{\pi} \mathrm{d} z \int_{-\pi}^{\pi} \frac{\mathrm{d} x \mathrm{e}^{i x}}{E-h(x, y, z)}, \\
& g_{2}(E)=G_{01 ; 03}^{0}(E)=\frac{1}{4 \pi^{3}} \int_{-\pi / 2}^{\pi / 2} \mathrm{~d} y \mathrm{e}^{i y} \int_{-\pi}^{\pi} \mathrm{d} z \mathrm{e}^{i z} \int_{-\pi}^{\pi} \frac{\mathrm{d} x}{E-h(x, y, z)}, \\
& g_{3}(E)=G_{01 ; 04}^{0}(E)=\frac{1}{4 \pi^{3}} \int_{-\pi / 2}^{\pi / 2} \mathrm{~d} y \int_{-\pi}^{\pi} \mathrm{d} z \mathrm{e}^{i z} \int_{-\pi}^{\pi} \frac{\mathrm{d} x \mathrm{e}^{i x}}{E-h(x, y, z)}
\end{aligned}
$$

The explicit representations of (4.15) to (4.18) are involved expressions of integrals of complete elliptic integrals of the first and the third kind, and are given in a separate paper [18].

\section{The Effect of Clusters on the Optical Properties of Vibrational Excitons in the Benzene Crystal}

In this section we shall adopt the conventional crystallographic classification of the benzene crystal primitive vector. We shall treat the $v_{11}$ vibrational exciton band $[13,19]$. Our host will be perdeuterobenzene and our guest benzene where [20] $\Delta=$ $=168 \mathrm{~cm}^{-1}$. We shall set

$$
\begin{aligned}
& \boldsymbol{a}_{1}=\boldsymbol{a} \\
& \boldsymbol{a}_{2}=\boldsymbol{c} \\
& \boldsymbol{a}_{3}=\boldsymbol{b} .
\end{aligned}
$$

In addition we shall use the notation given by Laufer and Kopelman [15] for the

\begin{tabular}{|c|c|c|c|}
\hline parameter set & $\begin{array}{c}\mathrm{M}_{\mathrm{I}, \mathrm{II}} \\
\left(\mathrm{cm}^{-1}\right)\end{array}$ & $\begin{array}{c}\mathrm{M}_{\mathrm{I}, \mathrm{III}} \\
\left(\mathrm{cm}^{-1}\right)\end{array}$ & $\begin{array}{l}M_{\mathrm{I}, \mathrm{IV}} \\
\left(\mathrm{cm}^{-1}\right)\end{array}$ \\
\hline $\left.1[23]^{*}\right)$ & 0.7 & 4.1 & 0.9 \\
\hline $\left.2 \quad[24]^{*}\right)$ & -0.4 & 2.8 & 0.5 \\
\hline
\end{tabular}
interaction parameters $A, B, C$ :

$$
\left.\begin{array}{l}
A=M_{\mathrm{I}, \mathrm{II}} \\
B=M_{\mathrm{I}, \mathrm{IV}}, \\
C=M_{\mathrm{I}, \mathrm{III}}
\end{array}\right\}
$$

Table 1

Interaction parameters given by various investigators for the $\mathrm{C}_{6} \mathrm{H}_{6} v_{11}$ band

*) See also references $[10,13,16]$. 
Table 2

Cluster notations and probabilities for a square lattice topology and nearest neighbors interactions

\begin{tabular}{l|c|c}
\hline $\left.\mathrm{Z}_{n \alpha}^{*}\right)$ & $P_{n \alpha}$ & $\begin{array}{c}\text { cluster shape**) } \\
\text { (schematic) }\end{array}$ \\
\hline $\mathrm{Z}_{1}$ & $c(1-c)^{4}$ & $\times$ \\
$\mathrm{Z}_{2}$ & $2 c^{2}(1-c)^{6}$ & $\times$ \\
$\mathrm{Z}_{3(1)}$ & $2 c^{3}(1-c)^{8}$ & $\times$ \\
$\mathrm{Z}_{3(2)}$ & $4 c^{3}(1-c)^{7}$ & \\
$\mathrm{Z}_{41(1)}$ & $2 c^{4}(1-c)^{10}$ & \\
$\mathrm{Z}_{41(2)}$ & $8 c^{4}(1-c)^{9}$ & \\
$\mathrm{Z}_{41(3)}$ & $4 c^{4}(1-c)^{8}$ & \\
$\mathrm{Z}_{42}$ & $4 c^{4}(1-c)^{8}$ & \\
$\mathrm{Z}_{43}$ & $c^{4}(1-c)^{8}$ &
\end{tabular}

*) The additional notation in brackets for the $Z_{n \alpha}$ specifies shapes that correspond to the same eigenvalue sets given in Table 4.

**) $\mathrm{x}$ denotes a guest molecule belonging to a cluster. The lines connecting guest atoms correspond to a distance of $|(b+c) / 2|$.

where $M_{\mathrm{I}, \mathrm{II}}, M_{\mathrm{I}, \mathrm{III}}$ and $M_{\mathrm{I}, \mathrm{IV}}$ denote interactions between adjacent interchange equivalent molecules in the planes spanned by the pairs of $(\boldsymbol{a}, \boldsymbol{b}),(\boldsymbol{b}, \boldsymbol{c})$ and $(\boldsymbol{c}, \boldsymbol{a})$ vectors, respectively. The interaction $M_{\mathrm{I}, \mathrm{III}}$ is the strongest interaction (see Table 1 ), hence we shall limit this discussion to clusters in the $(\boldsymbol{b}, \boldsymbol{c})$ crystal plane. The notations,

\section{Table 3}

Intensities of the allowed transitions of $E^{v}(0)$ for the $\mathrm{C}_{6} \mathrm{H}_{6} \nu_{11}$ vibrational exciton band

\begin{tabular}{c|c|c}
\hline $\begin{array}{c}\text { exciton branch } \\
v\end{array}$ & $\begin{array}{c}\left|\mu^{v}\right|^{2} \\
\text { (in relative units) }\end{array}$ & $\begin{array}{c}\text { polarization } \\
\text { (crystal axis) }\end{array}$ \\
\hline 2 & 0.50 & $a$ \\
3 & 0.45 & $c$ \\
4 & 0.05 & $b$
\end{tabular}

*) See reference [13]. 
Table 4

Secular determinants for cluster states*)

\begin{tabular}{|c|c|c|c|c|c|}
\hline$Z_{n \alpha}$ & $D_{n \alpha}$ & $m=\mathbf{1}$ & $m==\mathbf{2}$ & $m=3$ & $m=4$ \\
\hline $\begin{array}{l}Z_{1} \\
Z_{2} \\
Z_{3} \\
Z_{41} \\
Z_{42} \\
Z_{43}\end{array}$ & $\begin{array}{l}u \\
u^{2}-v^{2} \\
u^{3}-2 u v^{2} \\
u^{4}-3 u^{2} v^{2}+v^{4} \\
u^{4}-3 u^{2} v^{2} \\
u^{4}-4 u^{2} v^{2}\end{array}$ & $\begin{array}{l}u \\
u-v \\
u \\
u+\gamma_{1} v \\
u \\
u\end{array}$ & $\begin{array}{l}u+v \\
u+2^{1 / 2} v \\
u+\gamma_{2} v \\
u \\
u\end{array}$ & $\begin{array}{l}u-2^{1 / 2} v \\
u-\gamma_{1} v \\
u+3^{1 / 2} v \\
u+2 v\end{array}$ & $\begin{array}{l}u-\gamma_{2} v \\
u-3^{1 / 2} v \\
u-2 v\end{array}$ \\
\hline
\end{tabular}

*) $u=1-\Delta g_{0}(E), v=-\Delta g_{3}(E), \gamma_{1}=\left(1+5^{1 / 2}\right) / 2, \gamma_{2}=\left(1-5^{1 / 2}\right) / 2$.

the topology, and the probabilities $P_{n}$ for clusters up to the forth order are given in Table 2.

There are three allowed optical transitions for $\boldsymbol{k}=\boldsymbol{0}$. Each transition is polarized along one of the crystal axes. The polarizations of these transitions are given in Table 3.

The density of states function for the clusters can be obtained utilizing (2.12). The roots $E_{m}(n \alpha)$ of (2.12) can be determined by solving the determinants of (2.11). The determinant $D_{n \alpha}$ and the roots $(m)$ corresponding to the various factors of $D_{n \alpha}$ are in Table 4.

The optical properties related to the clusters for the various polarizations are given by the imaginary part of $T^{\nu}(E)$ of (3.7), which is given in terms of $\operatorname{Im} S^{\nu}(E)$, see (3.16). $P_{n \alpha}$ of $(3.16)$ is given in Table 2 whereas $\left.M_{n \alpha}^{(v, r)}(E) / D_{n \alpha}^{(\nu, r)}(E)\right)$ is displayed in Table 5.

It should be noted that our cluster states are given within the framework of a twodimensional model. Hence, the ratio

$$
R_{i}^{(m)}=\frac{M_{n \alpha}^{(\nu, r)}}{D_{n \alpha}^{(p, r)}}
$$

Table 5

$R_{i}^{(m)}$ parameters (equation (5.3)) for the optical properties of the cluster states*)

\begin{tabular}{|c|c|c|c|c|}
\hline$Z_{n \alpha}$ & \multicolumn{2}{|l|}{$R_{1}^{(m)}$} & \multicolumn{2}{|l|}{$R_{2}^{(m)}$} \\
\hline $\mathrm{Z}_{1}$ & $R_{1}^{(1)}=1 / u$ & & $R_{2}^{(1)}=1 / u$ & \\
\hline $\mathrm{Z}_{2}$ & $R_{1}^{(2)}=2 /(u+v), R_{1}^{(1)}=0$ & $R_{1}^{(1)}=0$ & $R_{2}^{(1)}=2 /(u-v)$ & $R_{2}^{(2)}=0$ \\
\hline$Z_{3}$ & $\begin{aligned} R_{1}^{(m)}= & 4 /(u-v) /\left(u^{2}-2 v^{2}\right) ; \\
& m=2,3\end{aligned}$ & $R_{1}^{(1)}=0$ & $\begin{aligned} R_{2}^{(m)}= & 4 /(u+v) /\left(u^{2}-2 v^{2}\right) \\
& m=2,3\end{aligned}$ & $R_{2}^{(1)}=0$ \\
\hline $\mathrm{Z}_{41}$ & $\begin{aligned} R_{1}^{(m)}= & (4 u-2 v) / \\
& \left(u^{2}+u v-v^{2}\right) \\
& m=1,2\end{aligned}$ & $\begin{array}{l}R_{1}^{(m)}=0 \\
m=3,4\end{array}$ & $\begin{aligned} R_{2}^{(m)}= & (4 u+2 v) / \\
& \left(u^{2}-u v-v^{2}\right) \\
& m=3,4\end{aligned}$ & $\begin{array}{l}R_{2}^{(m)}=0 \\
m=1,2\end{array}$ \\
\hline$Z_{45}$ & $\begin{aligned} R_{1}^{(m)}= & (4 u-6 v) /\left(u^{2}-3 v^{2}\right) ; \\
& m=3,4\end{aligned}$ & $\begin{array}{l}R_{1}^{(m)}=0 \\
m=1,2\end{array}$ & $\begin{aligned} R_{2}^{(m)}= & (4 u+6 v) /\left(u^{2}-3 v^{2}\right) \\
& m=3,4\end{aligned}$ & $\begin{array}{l}R_{2}^{(m)}=0 \\
m=1,2\end{array}$ \\
\hline$Z_{43}$ & $R_{1}^{(3)}=4 /(u+2 v)$ & $\begin{array}{l}R_{2}^{(m)}=0 \\
m=1,2,4\end{array}$ & $R_{2}^{(4)}=4 /(u-2 v)$ & $\begin{array}{l}R_{2}^{(4)}=0 \\
m=1,2,3\end{array}$ \\
\hline
\end{tabular}

*) See footnote Table 4 for $u$ and $v$. 
corresponds only to two distinct solutions for the three allowed polarizations. In (5.3) $m$ stands for the $E_{m}^{(n \alpha)}$ eigenvalue of the $Z_{n \alpha}$ cluster, and $i=1$ for $v=2,3$ whereas $i=2$ for $v=4$.

\section{Numerical Results}

The Green's functions of (4.15) to (4.18) are displayed in Fig. 1, utilizing parameter set No. 1 of Table 1 . It is apparent from Fig. 1 that $g_{3}(E)$, which corresponds to the largest interaction $M_{\mathrm{I}, \mathrm{III}}$, is larger in absolute value than $g_{1}(E)$ and $g_{2}(E)$ which correspond to the smaller interactions $M_{\mathrm{I}, \mathrm{II}}$ and $M_{\mathrm{I}, \mathrm{IV}}$, respectively, for $E$ values outside the exciton host band; i.e. $-17.2>E>22.8$. As the perturbation strength increases, the difference between $g_{3}(E)$ and both $g_{1}(E)$ and $g_{2}(E)$ increases for the appropriate eigenvalues $E$.

The relative intensities given by $\operatorname{Im} T^{v}(E)$ (see (3.7)) of the various cluster lines corresponding to parameter set No. 2 Table 1 (up to the tetramers) are displayed in Fig. 2. The pertinent feature of the absorption lines is that strong lines are located below the monomer line for $v=2,3$ as shown ${ }^{4}$ ) in the left portion of Fig. 2 (for $\operatorname{Im} T^{(2)}$ ), whereas for $v=4$ the strong lines are above the monomer line. Results given in Fig. 3 correspond to the unpolarized absorption $\operatorname{Im} T$ of the $v_{11}$ vibrational exciton (sum of the three polarized components). The unpolarized spectrum is very similar to the $\nu=2$ spectrum (and $v=3$ ), because of the relative strength of these two transitions. On the other hand, the density of states $\varrho$ is symmetrical with respect to the monomer line. In addition, the monomer line has contributions from the trimers and tetramers. In contrast, the monomer optical line has very little
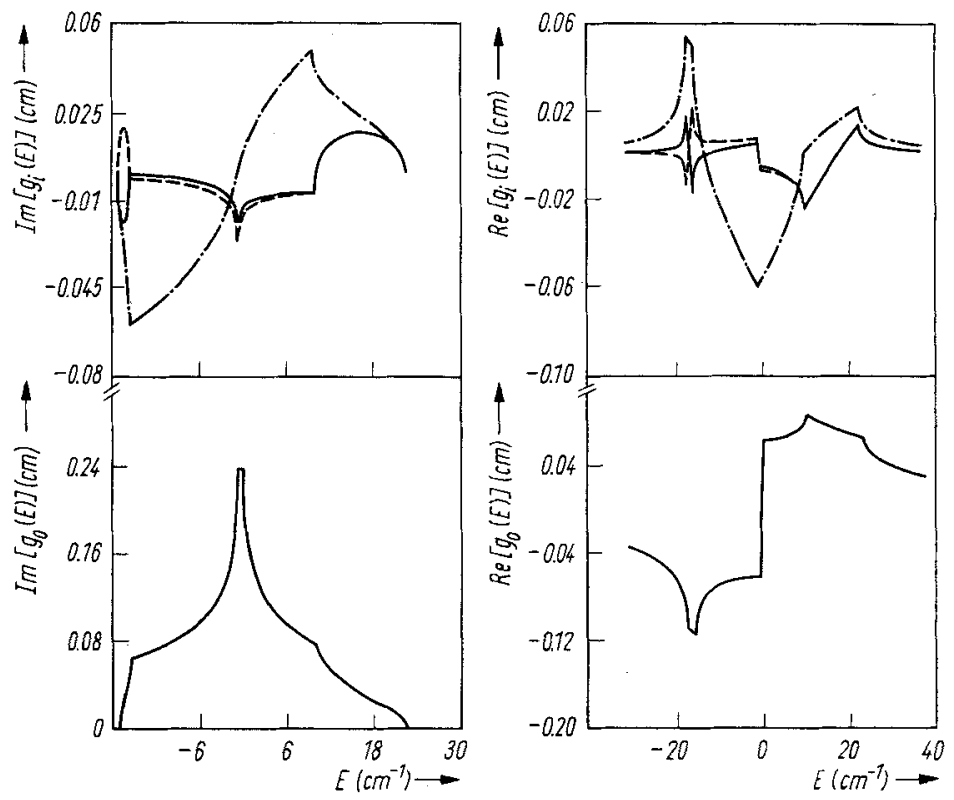

Fig. 1. Real and imaginary parts of the Green's functions matrix element $g_{i}(E)$. Bottom figures denote diagonal element $g_{0}(E)$. Top figures denote off-diagonal elements $g_{i}(E)$ : $---g_{1}(E)$, $g_{2}(E),-\cdots g_{3}(E)$. The Green's functions are given for parameter set No. 1 of Table 1

${ }^{4}$ ) The $v=3$ lines are not shown because they resemble to a very large extent the $v=2$ lines. 


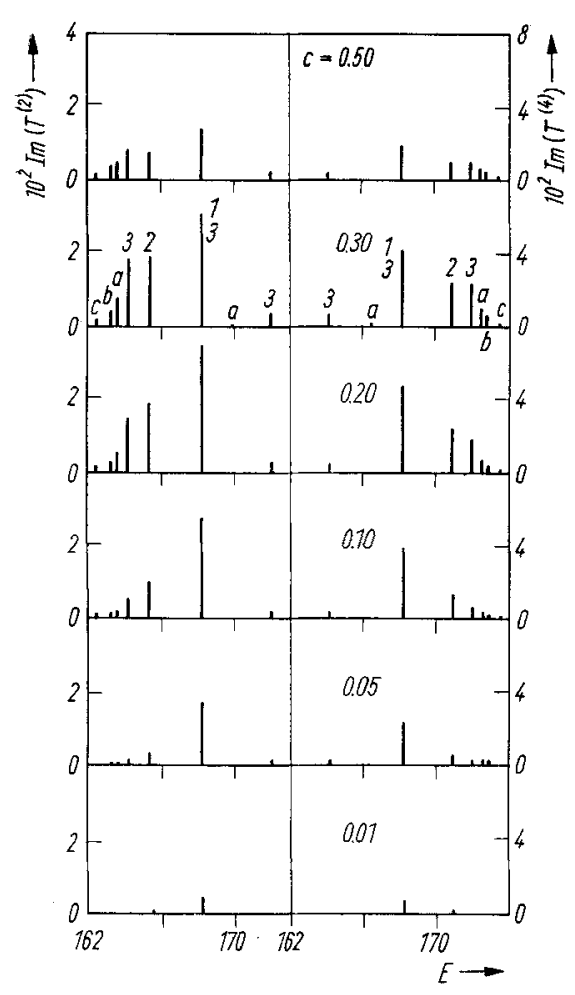

Fig. 2

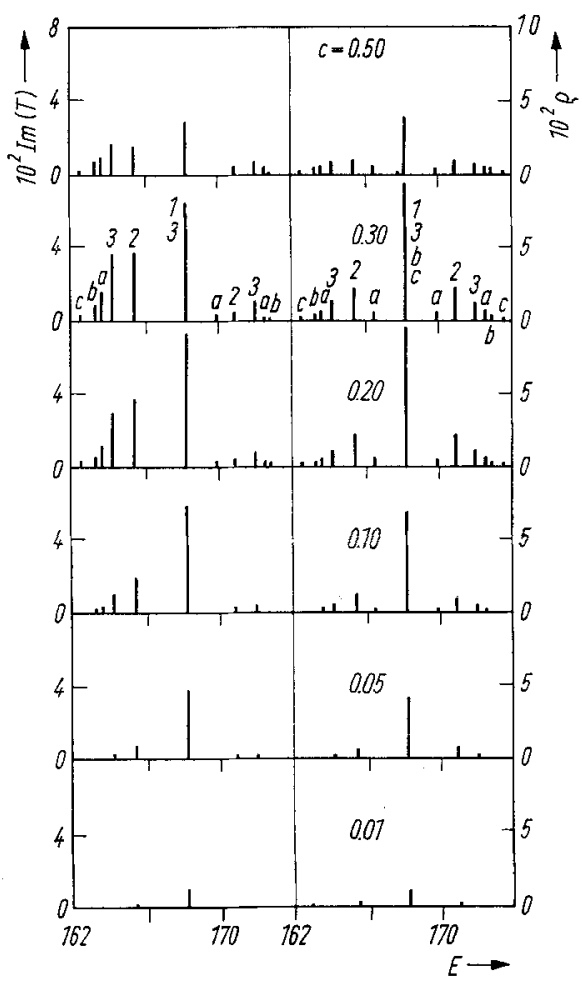

Fig. 3

Fig. 2. Relative optical absorptions ((see (3.7) and (3.16)), for exciton branches $y=2$ (a polarized) and $\nu=4$ ( $b$ polarized) given in Table 3, with parameter set No. 2 given in Table 1, and perturbation $\Delta=168 \mathrm{~cm}^{-1}$. The absorption lines are given for the clusters listed in Table 2 . Lines a, b, and $c$ correspond to clusters $Z_{41}, Z_{42}$ and $Z_{43}$, respectively. Concentrations $(C)$ are in mole fractions

Fig. 3. Unpolarized absorption $\operatorname{Im}(T)$ and density of states $\varrho$ for parameter set and perturbation as in Fig. 2

contribution from the higher clusters, and the spectrum is very much "monomeric" in nature. The dependence of the intensities of the cluster lines on the concentration exhibits first an increase with concentration for all species, then goes through a maximum at some concentration and then decreases. It should be noted that throughout the concentration range in Fig. 2 and 3 the monomer lines are relatively strong even for $c=0.5$. At this concentration we can observe a marked reduction in intensity for all species, since we are approaching the percolation concentration $[2,6]$ for the twodimensional square lattice: $c_{\mathrm{p}}=0.59$. At the percolation concentration we begin to obtain contributions to our spectra from the infinite cluster. It should be noted that our scheme (including only monomers, dimers, trimers and tetramers) gives over $99.9 \%$ of the total guest spectra for $c=0.01$, but diminishes to about $80 \%$ at $c=0.2$. At $c=0.5$ the monomers, dimers, trimers and tetramers account only for $12 \%$ of the guest optical spectra. The effect of varying the pure erystal band-width is given in Fig. 4. The narrowing of the band yields cluster lines with reduced spacing. However, the relative intensities of the cluster lines do not change as the band-width changes, as can be expected from (3.16). 

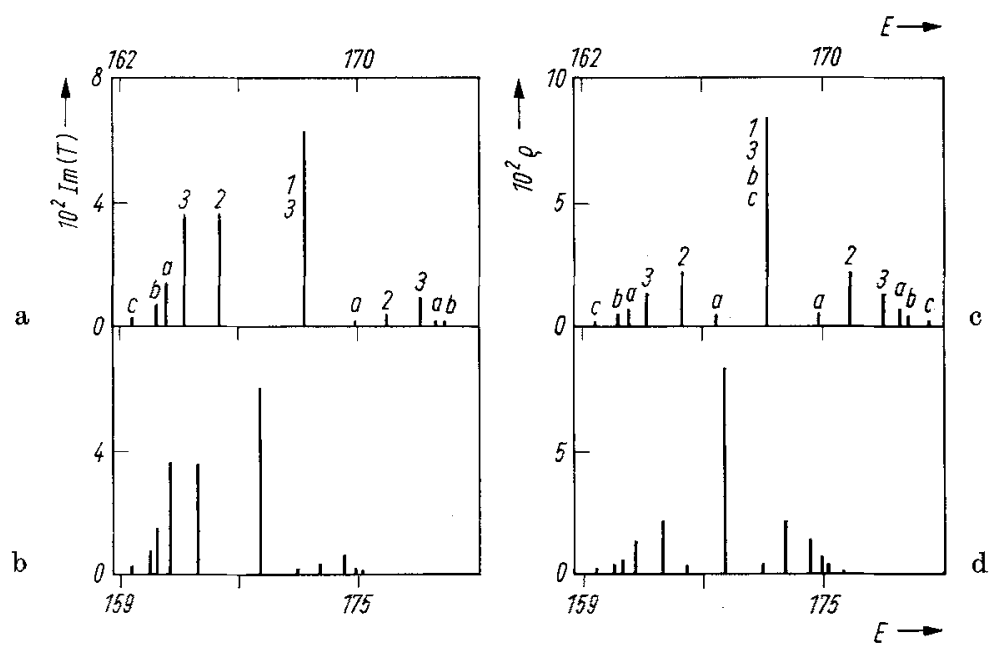

Fig. 4. Comparison of the optical absorption $\operatorname{Im}(T)$ and the density of states $\varrho$ for two different sets of interaction parameters. a) and c) correspond to parameter set 2 of Table 1 , whereas b) and d) correspond to set 1 . The perturbation is: $\Delta=168 \mathrm{~cm}^{-1}$. The notation for the lines are the same as in Fig. 1. The lines on the bottom figures b) and d) are in the same order as the lines in the top figures a) and c). Note the different $E$ scales. Here $C=0.3$.

\section{Conclusion}

In the previous sections we have been able to apply the cluster formalism for a twodimensional lattice. The results for the $\boldsymbol{v}_{\mathbf{1 1}}$ vibrational exciton are in qualitative agreement with the experimental data given by Hall [21]. The most noticeable resemblance between the theoretical and experimental data is the asymmetry in the optical lines, as well as the dominance of the monomer line bellow $c=0.5$. In a coming [22] experimental paper a more detailed comparison between theory and experiment will be given for the $\nu_{11}$ vibrational exciton. Although the theory given above was applied to a relatively simple model, it can be used for more complex systems. If, in addition to the nearest neighbor interactions, next nearest neighbor interactions are considered then the lines due to nearest neighbor interactions will be split into sets of dense lines, close to the original line positions. Taking even farther interactions, we would get more splittings, leading to a net result of broadening of the original nearest neighbor lines. This process would bring us closer to the experimental results. The main difficulty with such a process is the complexity of the cluster structure [25, 26]. There would be many types of clusters even for the lower members (pentamers, hexamers, etc.). The Green's functions corresponding to a large number of intermolecular interactions would be very complicated and difficult to evaluate.

\section{References}

[1] V. L. Brodde and E. I. Rashba, Pure appl. Chem. 37, 21 (1974).

[2] R. Kopelman, in: Excited States, Vol. 2, Ed. E. C. Lim, Academic Press, Inc., 1975 (p. 33).

[3] Y. Onodera and Y. To yozawa, J. Phys. Soc. Japan 24, 341 (1968).

[4a] H.-K. Hove and G. W. Robinson, J. chem. Phys. 52, 825 (1970); 54, 1369 (1971).

[4 b] O. A. Dubovskir and Y. V. Konobeev, Soviet Phys. - Solid State 12, 321 (1970).

[4c] J. Hoshen and J. Jortwer, Chem. Phys. Letters 5, 351 (1970).

[5] J. Hoshen and J. Jortner, J. chem. Phys. 56, 933, 5550 (1972). 
[6] H.-K. Hong and R. Kopelman, J. chem. Phys. 55, 724, 3491, 5380 (1971); 57, 3888 (1972).

[7] H.K. Hong and R. Kopelman, XXVII. Symp. Molecular Structure and Spectroscopy, paper D5, Ohio State University, Columbus, Ohio 1972.

[8] F. W. Ochs and R. Kopelman, J. chem. Phys. 66, 1599 (1977).

F. W. Ocнs, Ph.D. Thesis, University of Michigan, 1974.

[9] J. Hoshen and J. Jortwer, J. chem. Phys. 56, 4138 (1972).

[10] S. D. Colson, R. Kopelmav, and G. W. Robinson, J. chem. Phys. 47, 27, 5462 (1967).

E. R. Bernstein, S. D. Colson, R. Kopblman, and G. W. Robinson, J. chem. Phys. 48, 5596 (1968).

[11] Y. A. Izyumov, Adv. Phys. 14, 569 (1965).

[12] R. Kopelman, J. chem. Phys. 47, 2631 (1967).

[13] R. Kopelman, J. chem. Phys. 47, 3227 (1967).

[14] S. D. Colson, D. M. Hanson, R. Kopelman, and G. W. Robinson, J. chem. Phys. 48, 2215 (1968).

[15] J. C. Laufer and R. Kopelman, J. chem. Phys. 53, 3674 (1970).

[16] R. Kopelman and J. C. Laufer, in: Electronic Density of States, Ed. L. H. Bennett, Nat. Bur. Standards spec. Publ. No. 323, U.S.G.P.O., Washington (D. C.) 1971 (p. 261).

[17] J. Hoshen, J. Jortner, and R. Kopelman, Chem. Phys. 10, 185 (1975).

[18] J. Hoshen and R. Kopelman, J. Math. Phys. (New York) 17, 2067 (1976).

[19] J. C. Ladper and R. Kopelman, J. ehem. Phys. 57, 3203 (1972).

[20] J. H. Callomon, T. M. Dunn, and I. M. Mills, Phil. Trans. Roy. Soc. (London) 259, 499 (1966).

[21] L. HALL, Ph.D. Thesis, State University of Iowa, 1961.

[22] R. LeSar and R. Kopelman, Cluster Spectra and Vibrational Exciton Percolation in Solid Benzene (to be published).

R. LeSAR and R. Kopelman, XXX. Symp. Molecular Structure and Spectroscopy, paper MH3, Ohio State University, Columbus, Ohio 1975.

[23] M. P. Marzocchi, H. Bonadeo, and G. Taddei, J. chem. Phys. 53, 867 (1970).

[24] E. R. Bernstein and G. W. Robinson, J. chem. Phys. 49, 4962 (1968).

[25] J. Hoshen and R. Kopelman, Phys. Rev. B 14, 3438 (1976).

[26] R. Kopelman, Topics in Applied Physics, Vol. 15: Radiationless Processes in Molecules and Condensed Phases, Ed. F. K. Fong, Springer-Verlag, Berlin 1976 (p. 298). 